\title{
Modeling of flowering time in mungbean with Approximate Bayesian Computation
}

\author{
Ageev A.Yu. ${ }^{1}$, Lee C.-R. ${ }^{2}$, Ting C.-T. ${ }^{2}$, Schafleitner R. ${ }^{3}$, Bishop-von Wettberg E. ${ }^{4}$, \\ Nuzhdin S.V. ${ }^{1,5}$, Samsonova M.G. ${ }^{1}$, Kozlov K.N. ${ }^{1 *}$ \\ ${ }^{1}$ Peter the Great St. Petersburg Polytechnic University, St. Petersburg, Russia \\ ${ }^{2}$ National Taiwan University, Taipei, Taiwan \\ ${ }^{3}$ World Vegetable Center, Tainan, Taiwan \\ ${ }^{4}$ Department of Plant and Soil Science, University of Vermont, Burlington, VT, USA \\ ${ }^{5}$ University of Southern California, Los Angeles, CA, USA \\ *email: kozlov_kn@spbstu.ru
}

Mungbean (Vigna radiata (L.) Wilczek) is an important legume in many temperate regions. Its flowering time is a critical trait to achieve crop efficiency. We used 778 samples of 296 genotypes from 18 countries from the mungbean WorldVeg minicore collection and Approximate Bayesian Computation to construct the new dynamic mathematical models for flowering time under control of daily values of maximum and minimum temperature, precipitation, day length and solar radiation. It was shown that 12 and $19 \%$ of the variation in flowering time is explained by the interaction of climatic factors with the country of origin or genotype respectively. Comparison of the influence of climatic factors on groups of plants originating from different countries or carrying different combinations of alleles showed statistically significant differences in most cases, which additionally confirms the influence of the country of origin and genotype on plant phenology. Model forecasts for future years till 2030 for Taiwan showed that although the flowering time decreases for all accessions, different groups respond differently to the same climate change scenarios, these differences are determined by different impacts of climatic factors. For the refined model, it was shown that an increase in the daily maximum temperature from 0 to 2 degrees in 0.25 increments can cause two types of reactions - a decrease in the flowering time observed in most samples, and its growth revealed for individual values of the maximum temperature perturbation in a group of samples, which can be caused by insufficient watering.

Acknowledgments: The research was funded by the Ministry of Science and Higher Education of Russia as part of World-class Research Center program: Advanced Digital Technologies (contract No. 075-15-2020-934 dated 17.11.2020). 